tinuous mapping from $S^{*}$ into Euclidean $x y z$ space. Then $T$ determines a (not necessarily simple) closed surface $S$. Define an index-function $n(x, y, z)$ as follows: if the point $(x, y, z)$ lies on $S$, then $n=0$; if $(x, y, z)$ does not lie on $S$, then $n$ is equal to the topological index of the point $(x, y, z)$ with respect to $S$. Then $n(x, y, z)$ vanishes outside of a sufficiently large sphere $K$. Define $V(S)$, the volume enclosed by $S$, as the integral of $|n(x, y, z)|$ in $K$ if this integral exists, and let $V(S)=\infty$ otherwise. The purpose of the paper is to establish the isoperimetric inequality $V(S)^{2} \leqq A(S)^{3} / 36 \pi$, where $A(S)$ is the Lebesgue area of $S$, as a generalization of previous results of Tonelli and Bonnesen. (Received May 29, 1946.)

\title{
Statistics and Probability
}

\section{Z. W. Birnbaum: Tshebysheff inequality for two dimensions.}

For independent random variables $X, Y$ with expectations zero and variances $\sigma_{X}^{2}, \sigma_{Y}^{2}$ the trivial inequality $P\left(X^{2}+Y^{2} \geqq T^{2}\right) \leqq\left(\sigma_{X}^{2}+\sigma_{Y}^{2}\right) / T^{2}$ is replaced by a sharp inequality. (Received April 5, 1946.)

\section{Mark Kac: $A$ discussion of the Ehrenfest model. Preliminary} report.

A particle moves along a straight line in steps $\Delta$, the duration of each step being $\tau$. The probabilities that the particle at $k \Delta$ will move to the right or left are $(1 / 2)(1-k / R)$ and $(1 / 2)(1+k / R)$ respectively. $R$ and $k$ are integers and $|k| \leqq R$. M. C. Wang and G. E. Uhlenbeck in their paper On the theory of Brownian motion. II (Review of Modern Physics vol. 17 (1945) pp. 323-342) discuss this random walk problem and state several unsolved problems. In answer to some of the questions raised the following results are obtained: Let $(1-z)^{R-j}(1+z)^{R+j}=\sum C_{k}^{(j)} z^{k}(j$ an integer), then the probability $P(n, m \mid s)$ that a particle starting from $n \Delta$ will come to $m \Delta$ after time $t=s \tau$ is equal to $2^{-2 R}(-1)^{R+n} \sum(i / R)^{8} C_{R+j}^{(-n)} C_{R+m}^{(j)}$, where the summation is extended over all $j$ such that $|j| \leqq R$. Also, if $R$ is even the probability $P^{\prime}(n, 0 \mid s)$ that the particle starting from $n \Delta$ will come to 0 at $t=s \tau$ for the first time is calculated. For $n=0$ this gives a solution of the so-called recurrence time problem first studied on simpler models by Smoluchowski. Through a limiting process in which $\tau \rightarrow 0, \Delta \rightarrow 0, \Delta^{2} / 2 \tau \rightarrow D, 1 / R \tau \rightarrow \beta$, $n \Delta \rightarrow x_{0}, m \Delta \rightarrow x, s \tau=t$, one is led to fundamental distributions concerning the velocity of a free Brownian particle. In particular, $P(n, m \mid s)$ approaches the well known Ornstein-Uhlenbeck distribution. (Received May 23, 1946.)

\section{Howard Levene: $A$ test of randomness in two dimensions.}

A square of side $N$ is divided into $N^{2}$ unit cells, and each cell takes on the characteristics $A$ or $B$ with probabilities $p$ and $q=1-p$ respectively, independently of the other cells. A cell is an "upper left corner" if it is $A$ and the cell above and cell to the left are not $A$. Let $V_{1}$ be the total number of upper left corners and let $V_{2}, V_{3}, V_{4}$ be the number of similarly defined upper right, lower right, and lower left corners respectively. Let $V=\left(V_{1}+V_{2}+V_{3}+V_{4}\right) / 4$. It is proved that $V$ is normally distributed in the limit with $E(V)=p(N q+p)^{2}$ and $\sigma^{2}(V)=N^{2} p q^{2}\left(2-10 p+22 p^{2}-13 p^{3}\right) / 2$. The conditional limit distribution of $V$, when $p$ is estimated from the data, and the limit distribution of a related quadratic form are also obtained. These statistics are in a sense a generalization of the run statistics used for testing randomness in one dimension. (Received May 28, 1946.) 